\title{
Search for Sub-eV Sterile Neutrinos at RENO
}

\author{
J. H. Choi, ${ }^{1}$ H. I. Jang, ${ }^{2}$ J. S. Jang, ${ }^{3}$ S. H. Jeon, ${ }^{4}$ K. K. Joo, ${ }^{5}$ K. Ju, ${ }^{6}$ D. E. Jung, ${ }^{4}$ J. G. Kim, ${ }^{4}$ J. H. Kim, ${ }^{4}$ J. Y. Kim, ${ }^{5}$ \\ S. B. Kim, ${ }^{4}$ S. Y. Kim, ${ }^{7}$ W. Kim, ${ }^{8}$ E. Kwon, ${ }^{4}$ D. H. Lee, ${ }^{4}$ H. G. Lee, ${ }^{7}$ I. T. Lim, ${ }^{5}$ D. H. Moon, ${ }^{5}$ M. Y. Pac, ${ }^{1}$ \\ H. Seo, ${ }^{7}$ J. W. Seo, ${ }^{4}$ C. D. Shin, ${ }^{5}$ B. S. Yang $\odot,{ }^{9}$ J. Yoo, ${ }^{9,6}$ S. G. Yoon, ${ }^{6}$ I. S. Yeo, ${ }^{5}$ and I. Yu ${ }^{4}$
}

(RENO Collaboration)

\author{
${ }^{1}$ Institute for High Energy Physics, Dongshin University, Naju 58245, Korea \\ ${ }^{2}$ Department of Fire Safety, Seoyeong University, Gwangju 61268, Korea \\ ${ }^{3}$ GIST College, Gwangju Institute of Science and Technology, Gwangju 61005, Korea \\ ${ }^{4}$ Department of Physics, Sungkyunkwan University, Suwon 16419, Korea \\ ${ }^{5}$ Institute for Universe and Elementary Particles, Chonnam National University, Gwangju 61186, Korea \\ ${ }^{6}$ Department of Physics, KAIST, Daejeon 34141, Korea \\ ${ }^{7}$ Department of Physics and Astronomy, Seoul National University, Seoul 08826, Korea \\ ${ }^{8}$ Department of Physics, Kyungpook National University, Daegu 41566, Korea \\ ${ }^{9}$ Institute for Basic Science, Daejeon 34047, Korea
}

(Received 16 June 2020; revised 8 October 2020; accepted 9 October 2020; published 6 November 2020)

\begin{abstract}
We report a search result for a light sterile neutrino oscillation with roughly 2200 live days of data in the RENO experiment. The search is performed by electron antineutrino $\left(\bar{\nu}_{e}\right)$ disappearance taking place between six $2.8 \mathrm{GW}_{\text {th }}$ reactors and two identical detectors located at $294 \mathrm{~m}$ (near) and $1383 \mathrm{~m}$ (far) from the center of the reactor array. A spectral comparison between near and far detectors can explore reactor $\bar{\nu}_{e}$ oscillations to a light sterile neutrino. An observed spectral difference is found to be consistent with that of the three-flavor oscillation model. This yields limits on $\sin ^{2} 2 \theta_{14}$ in the $10^{-4} \lesssim\left|\Delta m_{41}^{2}\right| \lesssim 0.5 \mathrm{eV}^{2}$ region, free from reactor $\bar{\nu}_{e}$ flux and spectrum uncertainties. The RENO result provides the most stringent limits on sterile neutrino mixing at $\left|\Delta m_{41}^{2}\right| \lesssim 0.002 \mathrm{eV}^{2}$ using the $\bar{\nu}_{e}$ disappearance channel.
\end{abstract}

DOI: $10.1103 /$ PhysRevLett.125.191801

There remain unknown properties of neutrinos even with impressive progress in neutrino physics. The existence of sterile neutrinos has been a mysterious and unsolved problem [1]. The discovery of sterile neutrinos would not only open a new window into particle physics beyond the standard model, it would also solve the longstanding problem of dark matter in the Universe. Almost all the experimental results indicate that the number of light neutrino species is consistent with only three flavors. However, some experimental results may not be explained by the three active flavor neutrino hypothesis and suggest an additional flavor of neutrino with a mass around $1 \mathrm{eV}$ [2-7].

An interesting motivation for investigating a sub-eV sterile neutrino comes from cosmological data. Recent Planck data [8] seem to rule out an additional neutrino

Published by the American Physical Society under the terms of the Creative Commons Attribution 4.0 International license. Further distribution of this work must maintain attribution to the author(s) and the published article's title, journal citation, and DOI. Funded by SCOAP ${ }^{3}$. species with a mass near $1 \mathrm{eV}$ assuming full thermalization in the early Universe. However, sterile neutrinos have played an important role in explaining the dark radiation excess and the preference for a hot dark matter component with mass in the sub-eV range [9].

Recently, a combined data analysis by the MINOS+ and Daya Bay Collaborations reported a null observation of the sterile neutrino oscillations in the sub-eV region [10]. Based on this report's significant implication, an independent search with distinct detectors and neutrino sources is desirable for a chance of discovery or verification. Currently, searches for the light sterile neutrino oscillation are possible only by reactor experiments having baselines of $\sim 1 \mathrm{~km}$. RENO has performed a sub-eV sterile neutrino search that relies on the comparison of spectra measured by two identical detectors at different locations in order to find a spectral modulation due to the oscillation. By employing RENO's energy calibration, background subtraction, event selection, and detector performance, this analysis provides an independent search with unique experimental uncertainties and a distinct reactor complex.

This Letter reports a search for a light sterile neutrino based on the $3+1$ neutrino hypothesis using more than $10^{6}$ 
reactor $\bar{\nu}_{e}$ interactions in the RENO experiment. According to this hypothesis, the survival probability for $\bar{\nu}_{e}$ with an energy $E$ and a distance $L$ is approximately given by [11]

$$
P_{\bar{\nu}_{e} \rightarrow \bar{\nu}_{e}} \approx 1-\sin ^{2} 2 \theta_{13} \sin ^{2} \Delta_{13}-\sin ^{2} 2 \theta_{14} \sin ^{2} \Delta_{41},
$$

where $\Delta_{i j} \equiv 1.267 \Delta m_{i j}^{2} L / E, \Delta m_{i j}^{2} \equiv m_{i}^{2}-m_{j}^{2}$ is the masssquared difference between the mass eigenstates. This indicates that the sterile neutrino oscillation with a mixing angle $\theta_{14}$ introduces an additional spectral distortion by a squared mass difference $\left|\Delta m_{41}^{2}\right|$. Thus, these oscillation parameters can be explored by a model independent spectral comparison of the reactor $\bar{\nu}_{e}$ disappearance between near and far detectors. In this Letter, RENO presents a result of the light sterile neutrino search in its sensitive region of $\left|\Delta m_{41}^{2}\right| \lesssim 0.5 \mathrm{eV}^{2}$.

The RENO experiment uses two identical near and far detectors located at 294 and $1383 \mathrm{~m}$, respectively, from the center of six reactor cores at the Hanbit Nuclear Power Plant Complex in Yonggwang. The near (far) underground detector has $120 \mathrm{~m}(450 \mathrm{~m})$ of water equivalent overburden. Six pressurized water reactors, each with maximum thermal output of $2.8 \mathrm{GW}_{\mathrm{th}}$, are situated in a linear array spanning $1.3 \mathrm{~km}$ with equal spacings. The reactor flux-weighted baseline is $410.6 \mathrm{~m}$ for the near detector and $1445.7 \mathrm{~m}$ for the far detector, respectively. The baseline distances between the detectors and reactors are measured to an accuracy or better than $10 \mathrm{~cm}$ using the Global Positioning System and the total station system.

Each RENO detector consists of a main inner detector filled with 16 tons of $0.1 \%$ gadolinium $(\mathrm{Gd})$ loaded liquid scintillator and an outer veto detector. A reactor $\bar{\nu}_{e}$ is detected through the inverse beta decay (IBD) reaction $\bar{\nu}_{e}+p \rightarrow e^{+}+n$. Backgrounds are efficiently removed by time coincidence between a prompt signal and a delayed signal from neutron capture on Gd. The prompt signal releases energy of $1.02 \mathrm{MeV}$ as two $\gamma$ rays from the positron annihilation in addition to the positron kinetic energy. The delayed signal produces several $\gamma$ rays with the total energy of $\sim 8 \mathrm{MeV}$. The details of the RENO detector are described in Refs. [12-16].

Because of various baselines between two detectors and six reactor cores, ranging from $300 \mathrm{~m}$ to nearly $1.5 \mathrm{~km}$ as shown in Table I, this search is sensitive to mixing between active and sterile neutrinos in the region of $10^{-4} \lesssim\left|\Delta m_{41}^{2}\right| \lesssim 0.5 \mathrm{eV}^{2}$. These mixing parameters can

TABLE I. Baselines of near and far detectors from the six reactor cores.

\begin{tabular}{lcccccc}
\hline \hline & \multicolumn{6}{c}{ Baselines (m) } \\
\cline { 2 - 7 } Detectors & R1 & R2 & R3 & R4 & R5 & R6 \\
\hline Near & 660 & 445 & 302 & 339 & 520 & 746 \\
Far & 1564 & 1461 & 1398 & 1380 & 1409 & 1483 \\
\hline \hline
\end{tabular}

produce an additional modulation in energy with a frequency different from the active neutrino oscillation.

This analysis uses roughly 2200 live days of data taken in the period between August 2011 and February 2018. Applying the IBD selection criteria yields 850666 (103 212) $\bar{\nu}_{e}$ candidate events with the energy of prompt event $\left(E_{p}\right)$ between 1.2 and $8.0 \mathrm{MeV}$ in the near (far) detector. The remaining backgrounds are either uncorrelated or correlated IBD candidates due to random association between the prompt and delayed events, fast neutrons, and $\beta$-n emitters from cosmic-muon induced ${ }^{9} \mathrm{Li} /{ }^{8} \mathrm{He}$ isotopes. The remaining background rates and spectral shapes are obtained from control data samples $[16,17]$. The background fraction for the near (far) detector is $2.0 \%$ $(4.8 \%)$. The $E_{p}$ energy scale is determined by various radioactive sources and neutron capture events. A nonlinear response of scintillation to the prompt energy due to a quenching effect and Cherenkov radiation is well understood by the energy calibration. The $E_{p}$ resolution in the range of 1 to $8 \mathrm{MeV}$ is $8 \%$ to $3 \%$. A detailed description of IBD event selection, their systematic uncertainties, and background estimation can be found in Refs. [12,16,17].

The uncertainty in the absolute energy scale is estimated to be $1.0 \%$ [17]. This sterile neutrino search based on the relative measurement of spectra at two identical detectors is almost insensitive to the uncertainty. On the other hand, the $E_{p}$ difference between the near and far detectors makes the largest contribution to the uncertainty associated with this analysis. The relative energy-scale difference is estimated by comparing near and far spectra of calibration data and is found to be less than $0.15 \%$ [17]. This search is rather insensitive to the rest of systematic uncertainties because of their relatively minimal energy dependence. The uncorrelated reactor-flux uncertainty is $0.9 \%$, the uncorrelated detection efficiency uncertainty is $0.24 \%$, and the background uncertainty is $5.61 \%$ and $3.26 \%$ for the far and near detectors, respectively. These uncertainties contribute to the error of a relative rate measurement but minimally to that of this relative spectral-shape analysis.

The finite sizes of the reactor cores and the antineutrino detectors, relevant to a search in the region of $\left|\Delta m^{2}\right| \sim 1 \mathrm{eV}^{2}$, have a negligible effect on the sterile neutrino search in RENO's sensitive region of $\left|\Delta m^{2}\right| \lesssim 0.5 \mathrm{eV}^{2}$. The expected rates and spectra of reactor $\bar{\nu}_{e}$ are calculated for the duration of physics data taking by taking into account the varying thermal powers, fission fractions of four fuel isotopes, energy release per fission, fission spectra, IBD cross sections, and detector response [17].

RENO's multiple reactors provide various baselines between the near and far detectors for exploring a sterile neutrino oscillation in a wide range of $\left|\Delta m_{41}^{2}\right|$ values. With the various baselines and energies of reactor neutrinos, a sensitivity study for an excluded parameter region is performed using an Asimov Monte Carlo method [18]. 


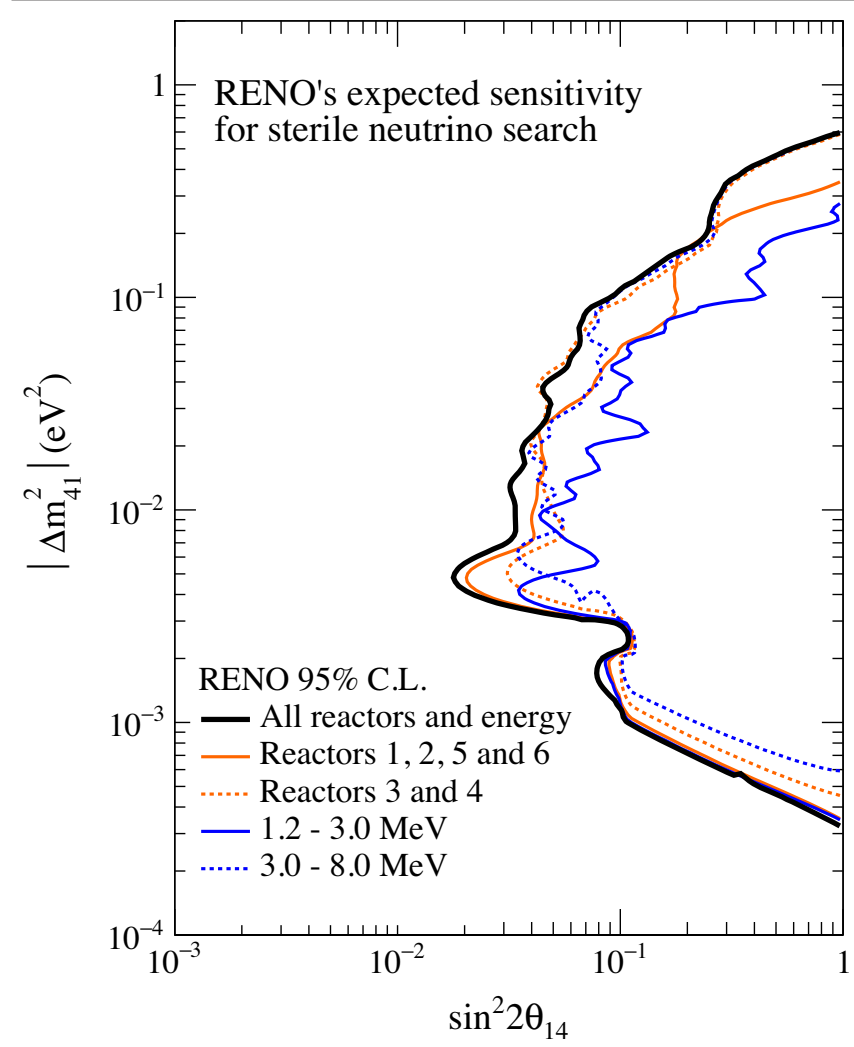

FIG. 1. Expected 95\% C.L. exclusion contours from sterile neutrino searches. The black solid contour represents an expected limit on $\bar{\nu}_{e}$ disappearance using RENO's 2200 days of data. The red solid (dotted) contour represents an exclusion sensitivity originating from a relatively long (short) baseline search. The blue solid (dotted) contour represents an exclusion sensitivity coming from a search in the $1.2-3.0 \mathrm{MeV}(3.0-8.0 \mathrm{MeV})$ region.

The sample is generated without statistical or systematic fluctuations assuming the three-neutrino hypothesis. Figure 1 shows an Asimov expected exclusion contour obtained from a search for a sterile neutrino oscillation by a far-to-near ratio method, which is described later. In the $10^{-4}<\left|\Delta m_{41}^{2}\right|<0.5 \mathrm{eV}^{2}$ region, a relative spectral distortion between the two detectors occurs and provides search sensitivity. The dip structure at $0.003 \mathrm{eV}^{2}$ is caused by a degenerate oscillation effect due to $\theta_{13}$ and $\theta_{14}$. In the $\left|\Delta m_{41}^{2}\right|<10^{-4} \mathrm{eV}^{2}$ region, an oscillation length becomes longer than the baseline distance between the two detectors and loses a search sensitivity. The sensitivity in the $0.01 \lesssim$ $\left|\Delta m_{41}^{2}\right| \lesssim 0.5 \mathrm{eV}^{2}\left(\left|\Delta m_{41}^{2}\right| \lesssim 0.01 \mathrm{eV}^{2}\right)$ region comes from the spectral comparison at relatively short (long) baselines between the two detectors or from the prompt energy above (below) $3 \mathrm{MeV}$. In the $\left|\Delta m_{41}^{2}\right| \gtrsim 0.5 \mathrm{eV}^{2}$ region, the far-tonear ratio method is unable to exclude any parameter region because of no relative spectral distortion between the two detectors. A rapid oscillation takes place before the near detector in the large $\left|\Delta m_{41}^{2}\right|$ region and generates no spectral distortion between the two detectors. However, comparison of their event rates becomes sensitive to exclude oscillation parameters.

This sterile neutrino search is based on comparison of observed spectra, with two identical detectors having different baselines, and thus reduces dependence on a reactor $\bar{\nu}_{e}$ flux and spectrum model. A sterile neutrino oscillation causes $\bar{\nu}_{e}$ disappearance according to Eq. (1) and produces relative spectral distortion between the near and far detectors. Figure 2 shows the ratio of the observed prompt energy spectrum at far detector and the threeneutrino best-fit prediction from the near detector spectrum [19]. The $3+1$ neutrino oscillation predictions are also shown for $\sin ^{2} 2 \theta_{14}=0.1$ and three $\left|\Delta m_{41}^{2}\right|$ values. The comparison between data and predictions demonstrates RENO's sensitivity of $\left|\Delta m_{41}^{2}\right| \lesssim 0.5 \mathrm{eV}^{2}$ in exploring a sterile neutrino oscillation. Because of the discrepancy of observed flux and spectra from the reactor $\bar{\nu}_{e}$ model prediction, this analysis employs the relative spectral distortion between identical near and far detectors. Moreover, the spectral ratio comparison cancels out common systematic uncertainties between the two identical detectors. The active and sterile oscillation parameters are determined by a fit to the measured far-to-near ratio of IBD prompt spectra in the same manner as the previous threeneutrino oscillation analysis [16]. To find the best fit, a $\chi^{2}$ with pull parameter terms of systematic uncertainties is constructed using the spectral ratio measurement and is minimized by varying the oscillation parameters and pull parameters as described in Ref. [16]:

$$
\begin{aligned}
\chi^{2}= & \sum_{i=1}^{N_{\text {bins }}} \frac{\left(O_{i}^{F / N}-T_{i}^{F / N}\right)^{2}}{U_{i}^{F / N}}+\sum_{d=N, F}\left(\frac{b^{d}}{\sigma_{\text {bkg }}^{d}}\right)^{2} \\
& +\sum_{r=1}^{6}\left(\frac{f_{r}}{\sigma_{\text {flux }}^{r}}\right)^{2}+\left(\frac{\epsilon}{\sigma_{\text {eff }}}\right)^{2}+\left(\frac{e}{\sigma_{\text {scale }}}\right)^{2},
\end{aligned}
$$

where $O_{i}^{F / N}$ and $T_{i}^{F / N}$ are the observed and expected far-tonear ratio of IBD events in the $i$ th $E_{p}$ bin, $U_{i}^{F / N}$ is the statistical uncertainty of $O_{i}^{F / N}$, and $O_{i}^{F / N}$ is the ratio of the spectra after background substraction given in Ref. [16]. The expected far-to-near ratio is calculated using reactor and detector information, including pull parameters $\left(b^{d}, f_{r}\right.$, $\epsilon$, and $e$ ). The systematic uncertainty sources are embedded by these pull parameters with associated systematic uncertainties $\left(\sigma_{\mathrm{bkg}}^{d}, \sigma_{\mathrm{flux}}^{r}, \sigma_{\mathrm{eff}}\right.$, and $\left.\sigma_{\text {scale }}\right)$. The details of pull terms and systematic uncertainties are described in Ref. [16]. The $\chi^{2}$ is minimized with respect to the pull parameters and the oscillation parameters.

The oscillation parameters of $\theta_{14}, \theta_{13}$, and $\left|\Delta m_{41}^{2}\right|$ are set as free. The rest of variables are constrained with other measurements: $\sin ^{2} 2 \theta_{12}=0.846 \pm 0.021, \Delta m_{21}^{2}=$ $(7.53 \pm 0.18) \times 10^{-5} \mathrm{eV}^{2}$, and $\left|\Delta m_{32}^{2}\right|=(2.444 \pm 0.034) \times$ $10^{-3} \mathrm{eV}^{2}$ [19]. However, the parameters of $\theta_{12}$ and $\Delta m_{21}^{2}$ 


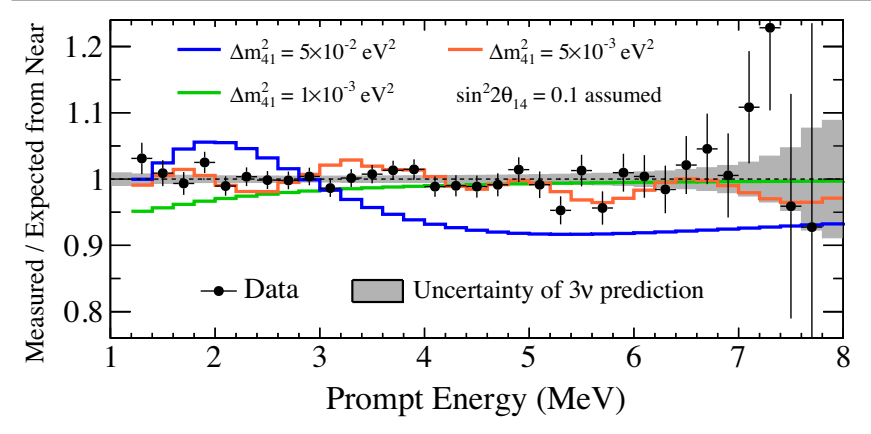

FIG. 2. Prompt energy spectra observed at far detector divided by the three-neutrino best-fit prediction from the near detector spectrum [19]. The gray band represents the statistical uncertainty of the near data and all the systematic uncertainties. Predictions with $\sin ^{2} 2 \theta_{14}=0.1$ and three $\left|\Delta m_{41}^{2}\right|$ representative values are also shown as the blue, red, and green curves.

are fixed because of their negligible effect on $\chi^{2}$. The parameter $\Delta m_{31}^{2}$ only is constrained by a pull term in the $\chi^{2}$. The normal mass ordering is assumed for both $\Delta m_{31}^{2}$ and $\Delta m_{41}^{2}$.

The minimum $\chi^{2}$ value for the $3+1$ neutrino hypothesis is $\chi_{4 \nu}^{2} / \mathrm{NDF}=46.4 / 65$, where $\mathrm{NDF}$ is the number of degrees of freedom. The value for the three-neutrino model with unconstrained $\left|\Delta m_{31}^{2}\right|$ is $\chi_{3 \nu}^{2} / \mathrm{NDF}=47.8 / 66$. The distribution of $\chi^{2}$ difference between the two hypotheses, $\Delta \chi^{2}=\chi_{3 \nu}^{2}-\chi_{4 \nu}^{2}$, is obtained from a number of simulated experiments with a statistical variation and their $\chi^{2}$ fits with systematic uncertainties taken into account. The $p$ value corresponding to the $\Delta \chi^{2}$ value is obtained to be 0.87 for $\Delta \chi^{2}=1.4$. This indicates the data are found to be consistent with the three-neutrino model and show no significant evidence for a sterile neutrino oscillation.

Exclusion limits in a parameter space of $\sin ^{2} 2 \theta_{14}$ and $\left|\Delta m_{41}^{2}\right|$ are set on sterile neutrino oscillation by a standard $\Delta \chi^{2}$ method [19]. For each parameter set of $\sin ^{2} 2 \theta_{14}$ and $\left|\Delta m_{41}^{2}\right|, \Delta \chi^{2}=\chi^{2}-\chi_{\min }^{2}$ is obtained, where $\chi_{\min }^{2}$ is the minimum $\chi^{2}$ out of all possible parameter sets. The $\chi^{2}$ of each parameter set is obtained by minimizing with varying $\theta_{13}$ and $\left|\Delta m_{31}^{2}\right|$. The parameter sets of $\sin ^{2} 2 \theta_{14}$ and $\left|\Delta m_{41}^{2}\right|$ are excluded at $95 \%$ confidence level if $\Delta \chi^{2}$ is greater than 5.99 [19]. Figure 3 shows an exclusion contour obtained from the RENO data. We repeat obtaining exclusion contours using the Gaussian $\mathrm{CL}_{s}$ method [20,21]. For each set of $\sin ^{2} 2 \theta_{14}$ and $\left|\Delta m_{41}^{2}\right|$, this method calculates $p$ values for the three-neutrino and $3+1$ neutrino hypotheses and determines a $\mathrm{CL}_{s}$ value from them. A 95\% C.L. exclusion region is obtained by requiring a condition of $\mathrm{CL}_{s} \leq 0.05$. The $\Delta \chi^{2}$ and Gaussian $\mathrm{CL}_{s}$ methods obtain 95\% C.L. contours of negligible difference within a statistical fluctuation.

In order to understand the validity of the data analysis, a number of pseudoexperiments are generated within statistical fluctuation and without the sterile neutrino hypothesis. Exclusion contours for the pseudoexperiments are obtained by the same $\Delta \chi^{2}$ method as described above by taking into account the systematic uncertainties. Figure 3 also shows an expected $1 \sigma$ band of 95\% C.L. exclusion contours due to a statistical fluctuation and its median. RENO's obtained exclusion contour is mostly contained in the $1 \sigma$ band.

The fluctuating behavior of the obtained exclusion contour in the region of $\left|\Delta m_{41}^{2}\right| \gtrsim 0.002 \mathrm{eV}^{2}$ comes from the finite size of the data sample. In the $\left|\Delta m_{41}^{2}\right| \lesssim$ $0.002 \mathrm{eV}^{2}$ region, the spectral distortion appears in the low energy range and gradually disappears. The data exclude a larger range of $\sin ^{2} 2 \theta_{14}$ values than the Asimov prediction in this $\left|\Delta m_{41}^{2}\right|$ region. The spectral deviation from the three-neutrino prediction at low energy happens to be minimal and obtains a more excluded region than the most probable expectation. According to pseudoexperiments, such an exclusion contour away from the expectation is estimated to have a probability of roughly $20 \%$. A dip structure at $\left|\Delta m_{41}^{2}\right| \sim 0.003 \mathrm{eV}^{2}$ as found in the Asimov study is observed due to an oscillation degeneracy

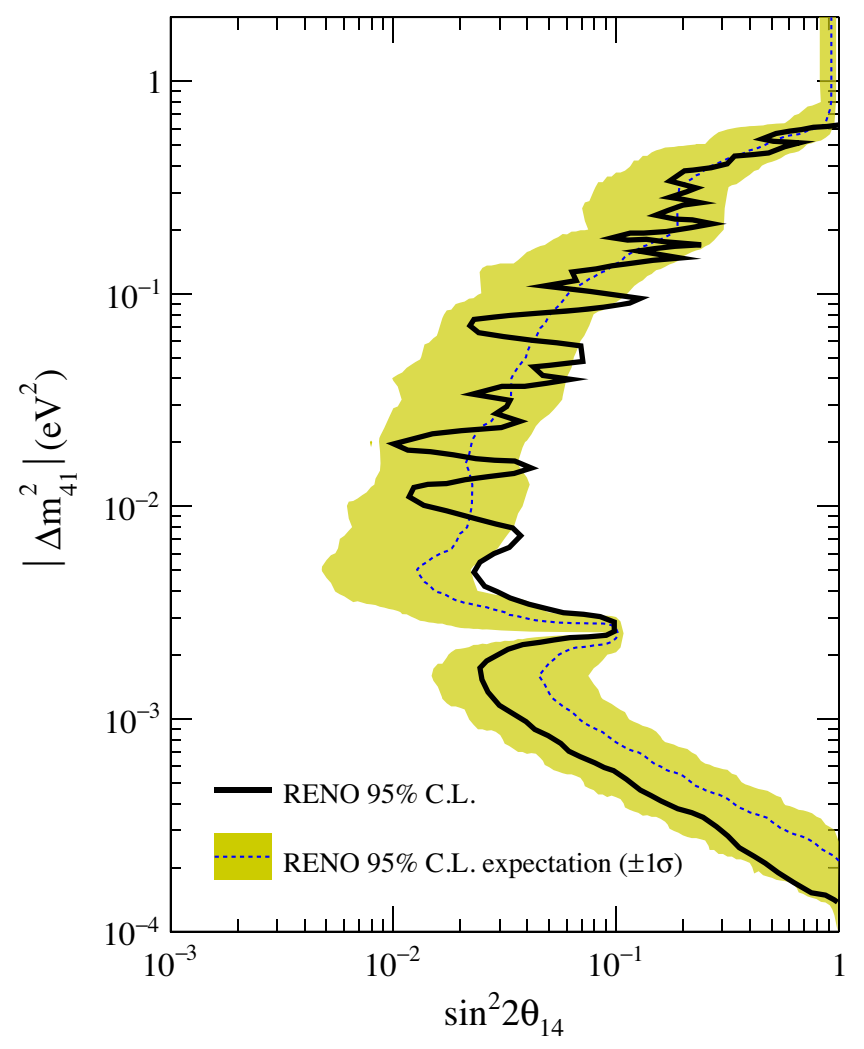

FIG. 3. RENO's 95\% C.L. exclusion contour for the sterile neutrino oscillation parameters of $\sin ^{2} 2 \theta_{14}$ and $\left|\Delta m_{41}^{2}\right|$. The black solid contour represents an excluded region obtained from spectral distortion between near and far detectors. The green shaded band represents expected $1 \sigma$ exclusion contours due to a statistical fluctuation. The blue dotted contour represents its median. The parameter region in the right side of the contours is excluded. 


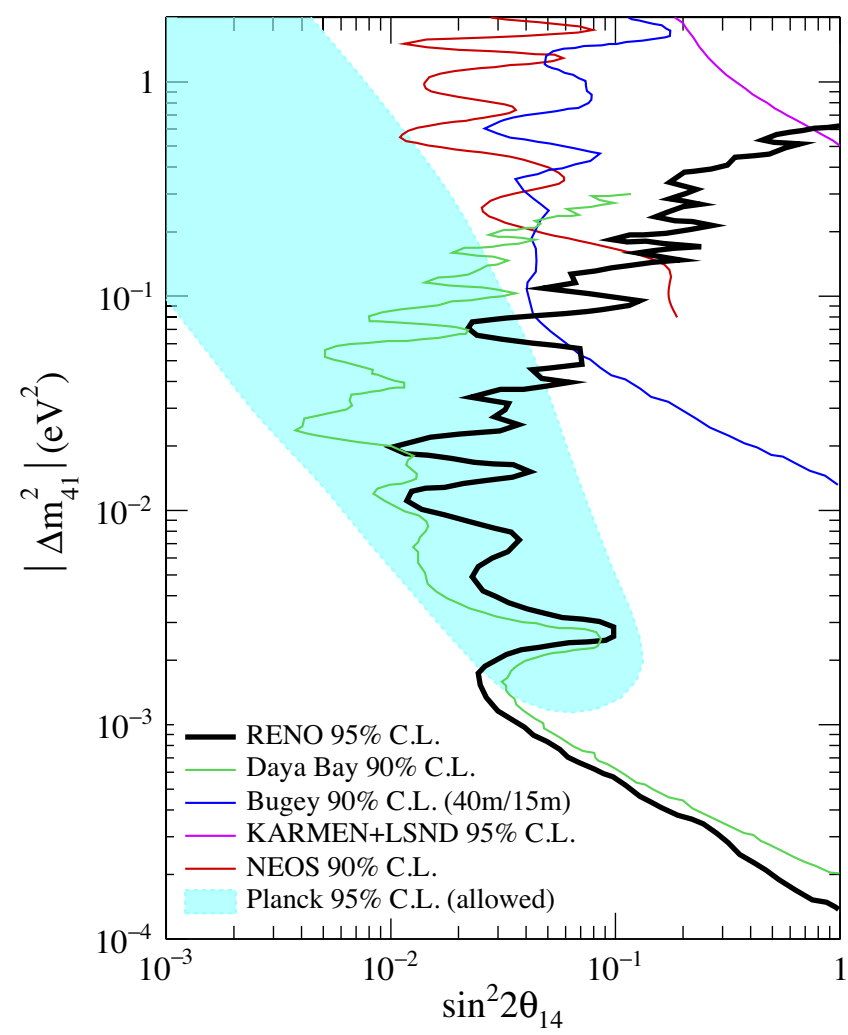

FIG. 4. Comparison of the exclusion limits. The right side of each contour indicates excluded region. The black solid line represents the $95 \%$ C.L. exclusion contour using spectral distortion between near and far spectra. For the comparison, Daya Bay's [10] 90\% C.L. (green), Bugey's [22] 90\% C.L. (blue), KARMEN + LSND [23] 95\% C.L. (magenta), and NEOS's [24] $90 \%$ C.L. (brown) limits on $\bar{\nu}_{e}$ disappearance are also shown. The blue shaded area represents a $95 \%$ C.L. allowed region from Planck data [25].

of $\theta_{13}$ and $\theta_{14}$. In the $\left|\Delta m_{41}^{2}\right| \gtrsim 0.5 \mathrm{eV}^{2}$ region, the spectral distortion due to the sterile neutrino oscillation is averaged out before the near detector and a search sensitivity is lost.

The limit of $\sin ^{2} 2 \theta_{14}$ is mostly determined by a statistical uncertainty, while the systematic uncertainties become considerable in the $\left|\Delta m_{41}^{2}\right| \lesssim 0.06 \mathrm{eV}^{2}$. The uncertainty of background $\left(\sigma_{\mathrm{bkg}}^{d}\right)$ is a dominant systematic source in the $0.003 \lesssim\left|\Delta m_{41}^{2}\right| \lesssim 0.06 \mathrm{eV}^{2}$ region, and the energyscale uncertainty $\left(\sigma_{\text {scale }}\right)$ is a major limiting factor in the $\left|\Delta m_{41}^{2}\right| \lesssim 0.008 \mathrm{eV}^{2}$ region. The uncertainties of flux $\left(\sigma_{\text {flux }}^{r}\right)$ and detection efficiency $\left(\sigma_{\text {eff }}\right)$ have negligible effect on this analysis.

Figure 4 shows exclusion contours obtained from the RENO data and other experiments as well as an allowed region from Planck data [10,22-25]. The RENO spectral comparison between the near and far detectors yields stringent limits on $\sin ^{2} 2 \theta_{14}$ in the $10^{-4} \lesssim\left|\Delta m_{41}^{2}\right| \lesssim$ $0.5 \mathrm{eV}^{2}$ region, while short baseline reactor neutrino experiments are sensitive to the $\left|\Delta m_{41}^{2}\right| \gtrsim 0.01 \mathrm{eV}^{2}$ region. RENO's $\sim 1 \mathrm{~km}$ baselines allow sensitivity to search for the
sub-eV sterile neutrino mixing. Combining the RENO result with those of other experiments can improve the sterile neutrino search sensitivity. More accurate short baseline reactor and accelerator neutrino experiments are desirable in order to probe the $\left|\Delta m_{41}^{2}\right|$ larger than $0.5 \mathrm{eV}^{2}$.

In summary, RENO reports results from a search for a sub-eV sterile neutrino oscillation in the $\bar{\nu}_{e}$ disappearance channel using 2200 days of data. No evidence for a sub-eV sterile neutrino oscillation is found using two identical detectors and thus yields a $95 \%$ C.L. limit on $\sin ^{2} 2 \theta_{14}$ in $10^{-4} \lesssim\left|\Delta m_{41}^{2}\right| \lesssim 0.5 \mathrm{eV}^{2}$. RENO obtains a significant excluded area of sub-eV sterile neutrino oscillation parameters by comparison of the measured IBD spectra using two identical detectors. The search minimizes dependence on reactor $\bar{\nu}_{e}$ flux and spectrum models. Based on a distinct reactor complex and RENO's unique systematic uncertainties, it provides an independent result for the sub-eV sterile neutrino oscillation. Combining it with the Daya Bay's result [10], one can firmly conclude there is no mixing between sub-eV sterile neutrino and $\bar{\nu}_{e}$ in the excluded parameter region. The RENO result provides the most stringent limits on sterile neutrino mixing at $\left|\Delta m_{41}^{2}\right|<$ $0.002 \mathrm{eV}^{2}$ using the $\bar{\nu}_{e}$ disappearance channel.

The RENO experiment is supported by the National Research Foundation of Korea (NRF), Grant Nos. 20090083526, 2019R1A2C3004955, and 2017R1A2B4011200 funded by the Korea Ministry of Science and ICT. Some of us have been supported by a fund from the BK21 of NRF and Institute for Basic Science (IBS-R017-D1-2020-a00/ IBS-R017-G1-2020-a00). We gratefully acknowledge the cooperation of the Hanbit Nuclear Power Site and the Korea Hydro and Nuclear Power Co., Ltd. (KHNP). We thank KISTI for providing computing and network resources through GSDC, and all the technical and administrative people who greatly helped in making this experiment possible.

[1] B. Pontecorvo, Neutrino experiments and the problem of conservation of leptonic charge, Zh. Eksp. Teor. Fiz. 53, 1717 (1967) [Sov. Phys. JETP 26, 984 (1968)], http://jetp.ac .ru/cgi-bin/dn/e_026_05_0984.pdf.

[2] C. Athanassopoulos, L. B. Auerbach, R. L. Burman, I. Cohen, D. O. Caldwell et al. (LSND Collaboration), Evidence for $\bar{\nu}_{\mu} \rightarrow \bar{\nu}_{e}$ Oscillations from the LSND Experiment at the Los Alamos Meson Physics Facility, Phys. Rev. Lett. 77, 3082 (1996).

[3] W. Hampel et al. (GALLEX Collaboration), Final results of the ${ }^{51} \mathrm{Cr}$ neutrino source experiments in GALLEX, Phys. Lett. B 420, 114 (1998).

[4] J. N. Abdurashitov, V. N. Gavrin, V. V. Gorbachev, P. P. Gurkina, T. V. Ibragimova et al. (SAGE Collaboration), Measurement of the solar neutrino capture rate with gallium metal. III. Results for the 2002-2007 data-taking period, Phys. Rev. C 80, 015807 (2009). 
[5] G. Mention, M. Fechner, T. Lasserre, T. A. Mueller, D. Lhuillier, M. Cribier, and A. Letourneau, The reactor antineutrino anomaly, Phys. Rev. D 83, 073006 (2011).

[6] A. A. Aguilar-Arevalo, B. C. Brown, L. Bugel, G. Cheng, E. D. Church et al. (MiniBooNE Collaboration), Improved Search for $\bar{\nu}_{\mu} \rightarrow \bar{\nu}_{e}$ Oscillations in the Miniboone Experiment, Phys. Rev. Lett. 110, 161801 (2013).

[7] C. Giunti and M. Laveder, Statistical significance of the gallium anomaly, Phys. Rev. C 83, 065504 (2011).

[8] P. A. R. Ade et al. (Planck Collaboration), Planck 2015 results-XIII. Cosmological parameters, Astron. Astrophys. 594, A13 (2016).

[9] S. Gariazzo, C. Giunti, and M. Laveder, Light sterile neutrinos in cosmology and short-baseline oscillation experiments, J. High Energy Phys. 11 (2013) 211.

[10] P. Adamson et al. (MINOS+, Daya Bay collaborations), Improved Constraints on Sterile Neutrino Mixing from Disappearance Searches in the MINOS, MINOS+, Daya Bay, and Bugey-3 Experiments, Phys. Rev. Lett. 125, 071801 (2020).

[11] A. Palazzo, Constraints on very light sterile neutrinos from $\theta_{13}$-sensitive reactor experiments, J. High Energy Phys. 10 (2013) 172.

[12] J. H. Choi, W. Q. Choi, Y. Choi, H. I. Jang, J. S. Jang et al. (RENO Collaboration), Observation of Energy and Baseline Dependent Reactor Antineutrino Disappearance in the RENO Experiment, Phys. Rev. Lett. 116, 211801 (2016).

[13] K. S. Park et al. (RENO Collaboration), Construction and properties of acrylic vessels in the reno detector, Nucl. Instrum. Methods Phys. Res., Sect. A 686, 91 (2012).

[14] J. S. Park et al. (RENO Collaboration), Production and optical properties of Gd-loaded liquid scintillator for the RENO neutrino detector, Nucl. Instrum. Methods Phys. Res., Sect. A 707, 45 (2013).

[15] K. J. Ma et al. (RENO Collaboration), Time and amplitude of afterpulse measured with a large size photomultiplier tube, Nucl. Instrum. Methods Phys. Res., Sect. A 629, 93 (2011).

[16] G. Bak, J. H. Choi, H. I. Jang, J. S. Jang, S. H. Jeon et al. (RENO Collaboration), Measurement of Reactor Antineutrino Oscillation Amplitude and Frequency at RENO, Phys. Rev. Lett. 121, 201801 (2018).

[17] S. H. Seo, W. Q. Choi, H. Seo, J. H. Choi, Y. Choi et al. (RENO Collaboration), Spectral measurement of the electron antineutrino oscillation amplitude and frequency using 500 live days of RENO data, Phys. Rev. D 98, 012002 (2018).

[18] G. Cowan, K. Cranmer, E. Gross, and O. Vitells, Asymptotic formulae for likelihood-based tests of new physics, Eur. Phys. J. C 71, 1554 (2011); Erratum, Eur. Phys. J. C 73, 2501 (2013).

[19] M. Tanabashi et al. (Particle Data Group), Review of particle physics, Phys. Rev. D 98, 030001 (2018).

[20] A. L. Read, Presentation of search results: The $\mathrm{CL}_{s}$ technique, J. Phys. G 28, 2693 (2002).

[21] X. Qian, A. Tan, J. J. Ling, Y. Nakajima, and C. Zhang, The Gaussian $\mathrm{CL}_{s}$ method for searches of new physics, Nucl. Instrum. Methods Phys. Res., Sect. A 827, 63 (2016).

[22] B. Achkar et al. (Bugey Collaboration), Search for neutrino oscillations at 15, 40 and 95 meters from a nuclear power reactor at bugey, Nucl. Phys. B434, 503 (1995).

[23] J. M. Conrad and M. H. Shaevitz, Limits on electron neutrino disappearance from the KARMEN and LSND $\nu_{e}$-carbon cross section data, Phys. Rev. D 85, 013017 (2012).

[24] Y. J. Ko, B. R. Kim, J. Y. Kim, B. Y. Han, C. H. Jang et al. (NEOS Collaboration), Sterile Neutrino Search at the NEOS Experiment, Phys. Rev. Lett. 118, 121802 (2017).

[25] A. M. Knee, D. Contreras, and D. Scott, Cosmological constraints on sterile neutrino oscillations from Planck, J. Cosmol. Astropart. Phys. 07 (2019) 039. 